\title{
Tornow, Siegfried, Abendland und Morgenland im Spiegel ihrer Sprachen. Ein kulturhistorischer Vergleich
}

Benoît Grévin

\section{OpenEdition}

\section{Journals}

Édition électronique

URL : http://journals.openedition.org/ifha/6581

DOI : 10.4000/ifha.6581

ISSN : 2198-8943

Éditeur

IFRA - Institut franco-allemand (sciences historiques et sociales)

Référence électronique

Benoît Grévin, «Tornow, Siegfried, Abendland und Morgenland im Spiegel ihrer Sprachen. Ein kulturhistorischer Vergleich », Revue de l'IFHA [En ligne], Date de recension, mis en ligne le 01 janvier 2011, consulté le 22 septembre 2020. URL : http://journals.openedition.org/ifha/6581 ; DOI : https:// doi.org/10.4000/ifha.6581

Ce document a été généré automatiquement le 22 septembre 2020.

(C)IFHA 


\title{
Tornow, Siegfried, Abendland und Morgenland im Spiegel ihrer Sprachen. Ein kulturhistorischer Vergleich
}

\author{
Benoît Grévin
}

1 L'essai de S.T. (Orient et Occident au miroir de leurs langues. Un essai d'histoire culturelle comparée) se propose de comparer l'évolution des cultures linguistiques de l'Occident latin, de l'Orient slavo-grec et de l'Islam classique (d'al-Andalus à la Transoxiane et aux Tatars de la Volga), depuis leur formation antique et médiévale jusqu'à nos jours. L'ampleur du programme impose nombre de restrictions, et l'auteur précise en introduction qu'il envisage la question sous l'angle des pratiques de l'écrit. Le plan est chronologique. Une introduction historique (chapitre 1. «Grundlagen », p. 15-47) précède un second chapitre examinant les langues « sacrales/sacrées » (« Die Sakralsprachen », p. 48-87). Le troisième chapitre est consacré à l'émergence médiévale des langues vulgaires («Die Volksprachen im Mittelalter », p. 91-115), le quatrième aux mouvements de retour aux sources scripturaires (chapitre 4. « Zu den Quellen », p. 116-133), le cinquième à la Réforme (« Die Reformation », p. 134-137), le sixième à l'Europe des nations (" Das Europa der Vaterländer », p. 138-145), le septième aux constructions politiques turques (« Die Türken kommen », p. 146-163), le huitième aux Lumières (" Die Aufklärung », p. 164-176), le neuvième au nationalisme (" Der Nationalismus », p. 177-199), le dixième à l'évolution moderne de cas particuliers à la charnière des trois zones comparées ("Zwischen Morgenland und Abenland », p. 200-232, exemples roumain, albanais, tatare, azéri et turc anatolien), le onzième au monde soviétique ("Der Sozialismus », p. 233-237). Une conclusion plaque la progression historico-linguistique ainsi dégagée sur trois modèles d'évolution historique (linéaire, cyclique et dialectique) qui expliqueraient les différences des trois aires (Schluss, p. 238-246).

2 Si certaines schématisations historiques (absence de possibilité d'insertion des Musulmans dans les États chrétiens médiévaux posée en principe, p. 38) sont excusables étant donné l'ampleur du champ concerné, on émettra quelques réserves 
plus sérieuses sur l'organisation d'une synthèse claire et généralement bien documentée. La moitié du livre est occupée par des introductions historiques, au détriment $\mathrm{du}$ sujet. Le choix de se concentrer sur les évolutions de longue durée du statut des langues écrites et leur montée ou maintien au sommet de la hiérarchie symbolique linguistique s'accompagne d'une tendance à présenter cette histoire en liaison avec le parcours de penseurs ou écrivains fameux qui évacue la réflexion sociolinguistique. On ne trouvera donc presque rien sur les dynamiques d'interaction linguistique à l'intérieur de sociétés multilingues aussi complexes que l'Angleterre médiévale (anglais/français/latin/celtes), l'Égypte des Mamelouks (arabe/copte/turc), l'Autriche moderne (Babel kakanienne...). Une analyse plus structurée du fonctionnement sociolinguistique de ces sociétés aurait peut-être permis de ne pas aboutir à une conclusion pour le moins frustrante. Reste, pour un lecteur spécialiste d'histoire occidentale ou islamique qui sera souvent déçu par le schématisme des développements concernant sa spécialité, l'intérêt des pages concernant le troisième angle " slavo-byzantin " du triangle, qui apportent de nombreux éléments effectivement dignes d'une réflexion comparatiste.

Benoît Grévin (CNRS, Laboratoire de médiévistique occidentale

de Paris) 